Article

\title{
Estimating 3D Leaf and Stem Shape of Nursery Paprika Plants by a Novel Multi-Camera Photography System
}

\author{
Yu Zhang, Poching Teng, Yo Shimizu, Fumiki Hosoi and Kenji Omasa *
}

Graduate School of Agricultural and Life Sciences, The University of Tokyo, 1-1-1, Yayoi, Bunkyo-ku, Tokyo 113-8657, Japan; 0491342004@mail.ecc.u-tokyo.ac.jp (Y.Z.); 8015764991@mail.ecc.u-tokyo.ac.jp (P.T.); ayosh@mail.ecc.u-tokyo.ac.jp (Y.S.); ahosoi@mail.ecc.u-tokyo.ac.jp (F.H.)

* Correspondence: aomasa@mail.ecc.u-tokyo.ac.jp; Tel.: +81-3-5841-5340; Fax: +81-3-5841-8175

Academic Editors: Changzhi Li, Roberto Gómez-García and José-María Muñoz-Ferreras Received: 12 April 2016; Accepted: 6 June 2016; Published: 14 June 2016

\begin{abstract}
For plant breeding and growth monitoring, accurate measurements of plant structure parameters are very crucial. We have, therefore, developed a high efficiency Multi-Camera Photography (MCP) system combining Multi-View Stereovision (MVS) with the Structure from Motion (SfM) algorithm. In this paper, we measured six variables of nursery paprika plants and investigated the accuracy of 3D models reconstructed from photos taken by four lens types at four different positions. The results demonstrated that error between the estimated and measured values was small, and the root-mean-square errors (RMSE) for leaf width/length and stem height/diameter were $1.65 \mathrm{~mm}\left(R^{2}=0.98\right)$ and $0.57 \mathrm{~mm}\left(R^{2}=0.99\right)$, respectively. The accuracies of the 3D model reconstruction of leaf and stem by a 28-mm lens at the first and third camera positions were the highest, and the number of reconstructed fine-scale 3D model shape surfaces of leaf and stem is the most. The results confirmed the practicability of our new method for the reconstruction of fine-scale plant model and accurate estimation of the plant parameters. They also displayed that our system is a good system for capturing high-resolution 3D images of nursery plants with high efficiency.
\end{abstract}

Keywords: 3D modeling; leaf; stem; accuracy; MCP system; stereovision; MVS; SfM

\section{Introduction}

For plant breeding and growth monitoring, accurate measurements of plant structure parameters and plant functioning are very crucial [1,2]. Over the past three decades, 2D imaging has been applied to a variety of aspects, ranging from structural analysis [3], stomata movements and photosynthetic diagnosis [4-6], herbicide control [7,8], disease detection [9] and yield estimation [10]. However, as 2D imaging is not robust against the occlusion of plant organs, such as overlapping leaves and stems and changing shapes during the measurement, most of these applications are either for large-scale analysis or for simple plant variables' measurements at the early growth stage [11]. Recently, 3D imaging technologies for the robust measurement of plant breeding and growth parameters have emerged and been applied for solving this problem owing to the advance of sensing technology $[2,12,13]$.

Among the applications of 3D imaging technology in agriculture, 3D model construction for plants $[2,14]$ and properties' estimation for plant growth $[15,16]$ are the most noticeable. Today, there are many commonly-known data acquisition methods for these applications, including active and passive methods. Among the active sensing technologies, scanning LiDARs (Light Detection and Ranging) are usually used for constructing accurate and detailed 3D models, but such systems are usually expensive and complex [14,17]. In our previous research, we have evaluated the performance of portable scanning LiDAR for estimating the leaf inclination angle distribution, leaf area density 
profiles, vertical plant area density, forest structure, water storage, etc. [2,16,17]. However, scanning LiDAR has the shortcomings of not only high cost and much time consumed, but also different sensor properties and robustness against background illumination [18]. Moreover, as leaf shapes are difficult to capture accurately if the leaves move because of air movement, the scanning LiDAR measurements must be performed without any disturbance of air movement [19]. Apart from the above active technologies, depth-sensing cameras [20,21] are more easily deployable and a less complicated mode of 3D data acquisition, as they enable acquiring image data in real time about texture or lighting conditions. However, although the cameras are low cost and easily applied, the captured data are difficult to directly apply because of their low resolution and high noise.

Among the passive sensing technologies, Shape-from- $X$ and stereovision are the most widespread methods of acquiring 3D data. They have been successfully applied in indoor and outdoor studies. For example, Omasa et al. [22] showed the 3D microscopic measurement of intact petunia seedlings for measuring shape and growth with a modified shape-from-focus method that incorporated a linear regression operator for 3D reconstruction. As for stereovision, it is also widely applied to reconstruct 3D models of plants [15] under indoor conditions. For example, some researchers [23] showed leaf wilt detection by stereovision. For outdoor imaging in a limited scope or at larger scales, there was also much work in the literature that introduced the accuracy of stereovision [24,25] and the classification of various plants with experimental variations of the environmental factors [26]. For example, this was applied for measuring outer leaf inclination angles of soybean plants and tracking their diurnal and nocturnal leaf movements [27]. Moreover, it was also used for guidance and navigation in the field [28]. In short, these applications were mostly conducted by using binocular stereovision. However, there are many limitations. Firstly, similar to 2D imaging technology, self-occlusion is still considered one of the limitations in stereovision in conducting various visual tasks, such as 3D scene reconstruction, target recognition, stereo matching, visual tracking, and so on [2,29]. Self-occlusion occurs when one part of an object is occluded by another part of the object from the camera's view, which will affect the results of the above tasks. Besides, stereovision is easily affected by wind or strong sunlight in outdoor conditions. Stereo matching and accuracy vary with the kind of algorithm used. The local matching algorithm is truly efficient, but less accurate than the global one, which could be, computationally, very expensive [30]. Finally, its performance is poor for close-range observation of surfaces because of the homogeneous surface texture, which produces pockets of missing depth information of the plants.

In general, according to the comparison of the above active and passive sensing technologies under indoor or outdoor illumination conditions, we can find out that the best approaches for 3D reconstruction are often focused on a particular application and cannot be easily adapted for conducting complex measurements. For stereovision, it can be a promising approach if we can improve its self-occlusion and accuracy problems. Thus, in this study, we applied a Multi-View Stereovision (MVS) method to design a novel image acquisition system with several low-cost digital cameras under indoor illumination for 3D reconstruction.

The MVS method originated as a natural improvement of the two-view stereo algorithms. Instead of capturing two photographs from two different viewpoints, MVS would capture more viewpoints in between to increase robustness, e.g., to image noise or surface texture [31-33]. As only one viewing perspective makes modeling difficult and some areas might not have been covered sufficiently, the images captured from different and overlapping views can effectively reconstruct a scene. Harwin and Lucieer [34] also suggest data collection from different perspectives. However, for determining the intrinsic camera parameters and the position of the corresponding points from uncalibrated images taken by several cameras, other techniques to reconstruct both camera positions and $3 \mathrm{D}$ points are necessary. A novel solution is proposed to improve surface representation and construct 3D plant models through automated feature extraction and key point matching across images based on the Structure from Motion (SfM) algorithm. SfM is a stereovision technique of simultaneously estimating camera positions and orientations and 3D scene structure from an unordered image dataset [35]. This algorithm can mitigate the self-occlusion problem in the stereovision method. 
Moreover, compared to scanning LiDAR and other methods, the cost of producing SfM point clouds is very low. Thus, there is a great potential in applying the SfM algorithm to reconstruct the 3D model. In fact, the SfM algorithm and related techniques have been introduced and developed in the last two decades. For example, the Scale-Invariant Feature Transform (SIFT) operator [36] was introduced to provide a potent illustration of characteristics in situ and allows significant characteristics in other perspectives to be contrasted and matched. Bundle adjustment was applied to generate a cluster of 3D coordinates of matching characteristics. The SfM algorithm was originally applied to many ground-based applications. It was also applied widely in reconstructing cultural and urban features for 3D modeling [37-39]. Recently, there were some geographic applications based on aerial platforms for outdoor features $[27,40]$. However, the SfM method for plants has been demonstrated to be more difficult than for other characteristics due to their more complicated and irregular structures [41-43]. The SfM algorithm has also some limitations in dealing with missing data. In this study, therefore, we have studied the combination of SfM and MVS algorithms for optimizing photo consistency and auto-calibration with high accuracy.

According to our investigation, no studies have specifically reported 3D vegetation modeling and the accuracy obtained using the SfM and MVS algorithms in indoor conditions, especially for nursery plants [44-47]. Consequently, this study is necessary to develop a low cost and high accuracy Multi-Camera Photography (MCP) system to generate a 3D model for nursery plants based on the MVS and SfM algorithms. The novelty of our method lies in the integration of SfM and MVS algorithms in indoor conditions for nursery plants. According to our method, we designed our MCP-SfM image acquisition system and demonstrated its feasibility and effectiveness through many experiments. Our method has solved some problems or limitations of other methods, such as high cost, self-occlusion, low accuracy, information missing, noises, and so on. Therefore, our proposed approach is innovative. Our objective is to estimate and compare plant parameters with different cameras and lenses for 3D imaging. We will introduce some metrics for the qualitative evaluation of 3D imaging and its accuracy for different lenses used for 3D shape reconstruction under four different positions. We will finally discuss the accuracy discrimination of leaf and stems properties, as well as the characteristics and limitations of the proposed system.

\section{Materials and Methods}

\subsection{Plant Material}

Paprika seedlings were grown in a combined black four-square nursery box ( $35.5 \mathrm{~cm}$ length $\times 28.0 \mathrm{~cm}$ width $\times 7.6 \mathrm{~cm}$ high) with 9 small pots in an environmentally-controlled growth chamber for over 3 weeks after sowing. The pots were filled with artificial soil (mixture of vermiculite and perlite, 2:1, v/v). Plants were watered every two days with a 1:1000 dilution of HYPONeX nutrient solution (HYPONeX Japan Corp., Ltd., Osaka, Japan). They were illuminated for $12 \mathrm{~h}$ each day by LEDs and fluorescence lamps at a photosynthesis photon flux density (PPFD) of $50 \mathrm{mmol} \cdot \mathrm{m}^{-2} \cdot \mathrm{s}^{-1}$. The PPFD ratio of red and blue light was $4: 1$. The air temperature was $26.5^{\circ} \mathrm{C}$ for $16 \mathrm{~h}$ in the day and $24{ }^{\circ} \mathrm{C}$ for $8 \mathrm{~h}$ at night. The relative humidity was $70 \%$ in the day and $90 \%$ at night. The intact nursery paprika plants were measured in the experiments.

\subsection{Multi-Camera Photography-Structure from Motion System}

According to the principle of MVS, the depth from images can be recovered by corresponding two or more perspectives of the same object. The result of this process is a 3D point cloud, in which each 3D point matches to a pixel in one of the photos. In previous studies [25-27], the binocular stereo method was usually applied to two photos captured with two cameras divided by a horizontal distance known as the "baseline". In our system design, 4 cameras are used to compose the MCP-SfM system for obtaining the 3D point cloud data of nursery paprika plants' structure. The distance between lens and plant is about $35 \mathrm{~cm}$. The MCP-SfM system is able to obtain a high resolution by calibrating the 
cameras and images corresponding the 3D point coordinates and locations in actual measurement scales. At the beginning, AF (Auto Focus) mode was used. Then, MF (Manual Focus) mode was used for capturing the clear photos after adjusting the focus. The exposure settings are ISO100-6400 before capturing the image; we have calibrated each camera for lens distortion compensation. Along with the conditions, the camera settings can provide optimal results.

Figure 1 illustrates a schematic view of the combined MCP-SfM system. Four high resolution cameras (5184 × 3456 pixels; Canon EOS Kiss X7, Canon Industrial Co., Ltd., Tokyo, Japan) with 4 lenses of different focal lengths $(24 \mathrm{~mm}, 28 \mathrm{~mm}, 35 \mathrm{~mm}, 50 \mathrm{~mm})$ were used for the 3D range acquisition of individual plants. The camera positions (No. 1 to 4 ) on the adjustable bracket were fixed on the same side at four different angles. The left of Figure 1 shows the directions of the lens at each camera position. Positions 1 to 4 were $45^{\circ}$ apart from each other in terms of vertical arrangement. In Position 3, the view direction of the camera was almost horizontal and can capture the entire object. The camera-to-leaf distance was between 35 and $40 \mathrm{~cm}$. In order to obtain a high resolution view of the leaf and stem, the distance between the camera lens and the plants was slightly different for each camera position.

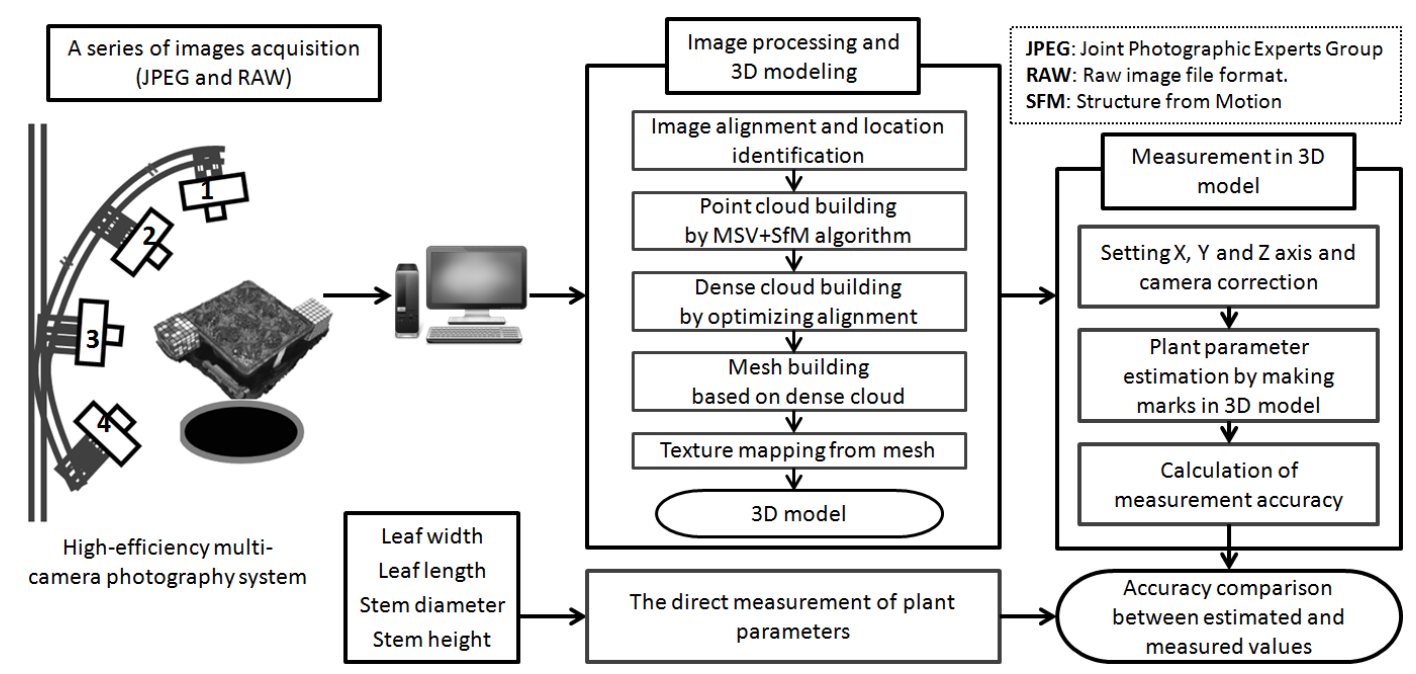

Figure 1. MCP-SfM system and the overall workflow for 3D modeling and measurement.

Image acquisition was done under indoor lighting conditions by varying the camera shutter speed. During the experiment, fluorescence lamps illuminate the entire plant in the four-square nursery box. The light adjustment exit was set so that the entire plant could be uniformly illuminated. In order to take the photos of the plant equably from a $360^{\circ}$ view, a turntable and timer with a stepper motor were used. During the measurement, the monitor and remote automatic shutter were also used for the photography of all cameras. The images are taken at 5184 horizontal 3456 vertical pixels per frame with 8-bit resolution in the system by a Canon EOS Kiss X7 camera equipped with a polarization filter (Kenko Circular PL 58 mm for reflection removal, Kenko Tokina Japan Co., Ltd., Tokyo, Japan). These image datasets will be input into PhotoScan (Agisoft LLC, St. Petersburg, Russia) for calibration processing and 3D model building on the computer (see Figure 1). Besides, as the data collection and processing need more time, we used an automatic shutter and turntable for continuous acquisition of images to improve the photography acquisition efficiency. In this way, we were able to intermittently capture one photo per $3 \mathrm{~s}$ (rotation time) by controlling the automatic turntable. In total, about $8 \mathrm{~min}$ were needed for a rotation each time.

\subsection{The MCP-SfM Approach}

The MCP-SfM approach can be used to obtain 3D data of objects and to calibrate a cluster of camera positions and 3D point locations for each camera track $[40,48,49]$. In order to understand the 
approach well, we can conduct an incremental approach, estimating for a pair of images at a time, rather than matching all of the images for all of the cameras and tracks at once. The first step was to estimate the camera positions and 3D points for a single pair of images. The original pair should have many feature correspondences, but also a baseline, so that the 3D point locations of the observed views are in the optimum state. Then, 3D point coordinates and locations are calculated for the selected image observed with the most tracks. The next step is to add the tracks observed by the next cameras into the optimization. A track can be augmented if it is observed by at least one other camera and if triangulating the tracks offers an optimum estimation of their locations. This process is reiterated, one image at a time, until there are no remaining images to be perceived by any of the generated 3D points. To optimize the objective function at each repetition, we use the bundle adjustment algorithm [50], which was employed by using an SfM platform, namely Microsoft PhotoSynth.

The resulting SfM consists of an accordant arbitrary coordinate system, which should be georeferenced and converted to real coordinates. In lab conditions, SfM datasets consist of a set of normal referenced coordinate point data with additional color information from the photographs, such as a Rubik's cube (see Figure 2). The key points generated from the SfM output can be located with $X, Y$ and $Z$ coordinates. Taking an example of using a pair of images by the SfM approach, the general process for solving the SfM problem is firstly to estimate the structure and motion up to a perspective transformation using the algebraic method or the factorization method proposed by Tomasi and Kanade [51]. For example, we need to estimate the $m 2 \times 4$ projection matrices $M_{i}$ (motion) and the $n 3 \mathrm{D}$ positions $S_{j}$ (structure) from the $m \times n$ 2D correspondences $s_{i j}$ (only allowing for translation and rotation between the cameras). This gives $2 m \times n$ equations with $8 m+3 n$ unknowns that can be solved using the algebraic method or the factorization method.

$$
s_{i j}=M_{i} S_{j}, \quad i=1, \ldots, \mathrm{m} ; j=1, \ldots, \mathrm{n}
$$

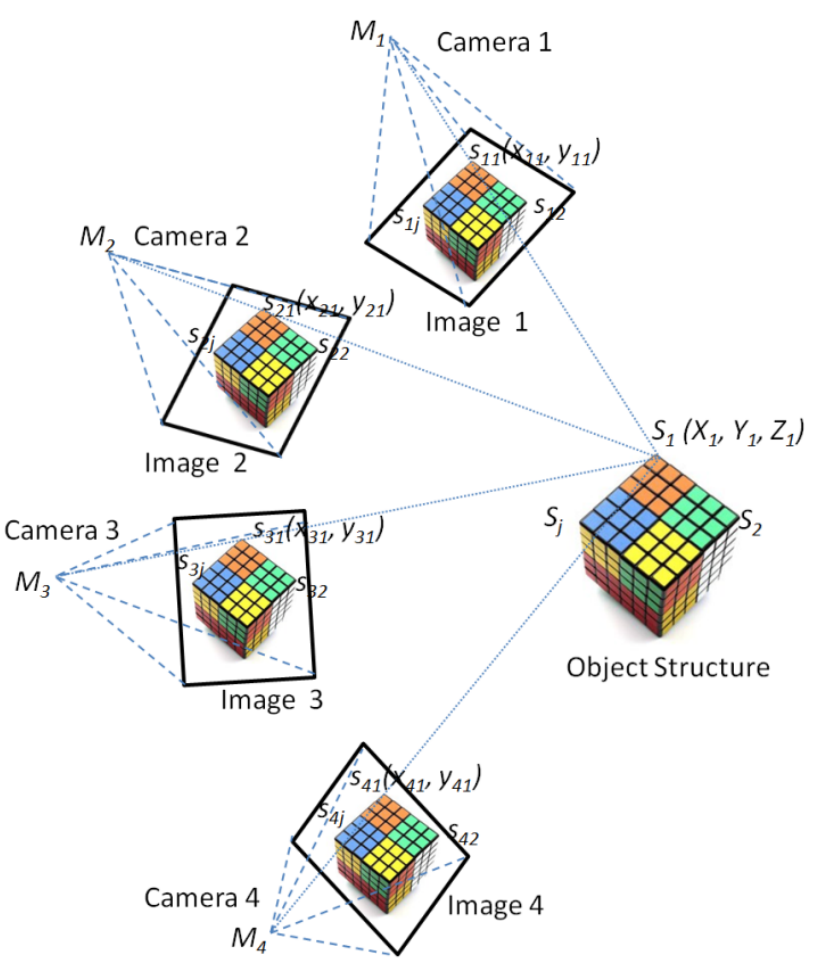

Figure 2. Methods for SfM for 3D model reconstruction.

Some measurements are computed with extractive points from the SfM datasets that are matched to parameter estimations (number of points, point heights, etc.). Then, we can convert from perspectives 
to metrics by auto-calibration and minimize reprojection errors by optimizing the location of 3D points and the camera by applying bundle adjustment.

Similarly, as for the MCP-SfM approach with multiple images (see Figure 2), we need to find a common scale so that multiple images can be combined. One method is to use the Perspective N-Point $(\mathrm{PnP})$ algorithm [52], where we try to solve for the position estimation of a new camera using the scene points we have already found. Typical SfM methods can robustly correspond characteristics in as many pairs of input photos as possible, so as to recover the estimations of the relatively accurate positions between photo pairs. Then, the bundle adjustment [53] calculates a maximum likelihood estimation of the camera positions and point positions, after initialization by a subset of the coupled estimations.

In this method, instead of increasing a single camera into the optimization, we increased several cameras at a time. As for selecting which cameras to increase, we originally need to get the camera with the most correspondence characteristics, $C$, to the subsistent 3D point clouds, then increase any camera with at least $75 \% C$ correspondences to the subsistent 3D point clouds. Generally, key point correspondence is very difficult when working with plant characteristics due to leaf sizes and gaps, repeating and inconsonant geometric structures, etc. [53]. Accordingly, some parts with few surface features may not be well captured; similarly, other parts with complicated geometric structures may not be well mapped or appear with radial distortion. Therefore, we implement the SfM algorithm to generate more precise point clouds and surface models at greater detail in spatial applications than the previous methods.

\subsection{D Model Processing of Nursery Plants}

Figure 3 shows the original photos of paprika plants taken by the MCP-SfM system. In Figure 3A, one of the original photos was taken by a $28-\mathrm{mm}$ lens at the 1 st camera position. The paprika canopy and leaf had a very high resolution. In Figure 3B, one of the original photos was taken by a 28-mm lens at the 3rd camera position. The paprika stem shows a very high resolution, as well as the references.
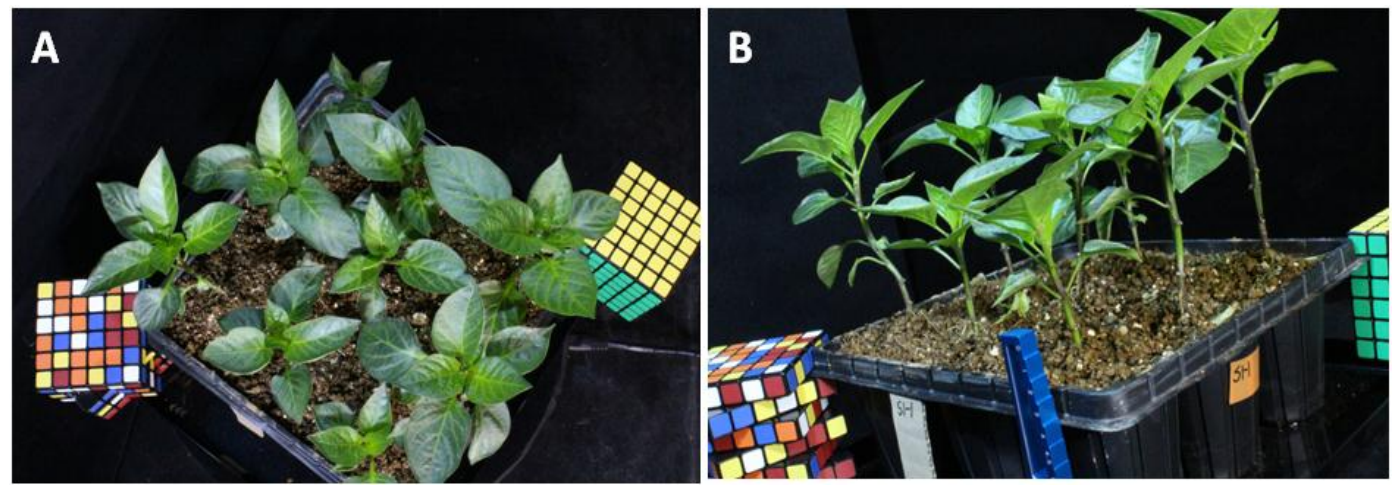

Figure 3. Original photos of paprika plants taken by the MCP-SfM system. (A) One of the original photos taken by a 28-mm lens at the 1st camera position; (B) One of the original photos taken by a 28-mm lens at the 3rd camera position.

Based on the MCP-SfM approach, the uncalibrated original image data were processed and calculated by PhotoScan. In summary, there are 5 steps of image processing during the 3D model rebuilding, as shown in the middle of Figure 1. As we know, the data taken from 4 different measuring views had individual coordinate systems. First of all, in order to make 4 cameras have the same 3D coordinate system, the image datasets obtained from 4 camera positions were co-registered into the orthogonal coordinates and aligned by location identification.

Because of the estimated matching points and camera positions, the datasets are registered through an accurate conversion. Then, the conversion was repeatedly extracted by selecting in turn matching points from the image dataset and getting the best translation and rotation matrices that minimize the error estimation on account of the distance between them. This process was conducted for all couples of the image dataset. Then, the colored point clouds of the stereo image data captured 
were produced with 4 calibrated stereo cameras by the MSV and SfM algorithms after calibrating the stereo camera system. These points are often located by $X, Y$ and $Z$ coordinates in the 3D coordinate system. The MSV algorithm starts from the calibrated stereo images and a set of tracks. For properly-calibrated stereo cameras, the non-distortion of stereo images is actually combined with rectification. The process computed disparity images from incoming stereo images using the block matching algorithm. The disparity images were generated using a local correlation algorithm based on Sum of Squared Differences (SSD). It was optimized for efficiency and has a number of validation steps to prune disparity. Note that the extracted 3D point clouds were entangled with redundancies, a large number of outliers and noise. Fortunately, the additional information provided by the calibrated images can be exploited to help mesh and surface reconstruction later. After exporting 3D point data, the dense point cloud model was reconstructed in four steps, which includes calculating the depth information for each camera, capturing dense point cloud correspondences and reconstructing the structure from motion and dense 3D point clouds. The dense point clouds could be edited and classified or exported to an external tool for further analysis. Then, the classified dense point clouds of the plant were transformed into polygon meshes. This procedure did some basic filtering to eliminate the most obviously irrelevant points. Thereafter, such polygon wireframe images of the plant allowed calculating the distance or area of leaves and stems.

Therefore, the 3D surface texture of leaves and stems was expressed, as the inconsistent triangle meshes depend especially on the alignment of the point cloud after noise exclusion. Moreover, triangulated meshing and surface reconstruction were applied to generate surface texture models. Laplacian smoothing was employed to smooth triangle meshing. A series of natural color photos captured after wireframing were matched on the surface model of the entire plant using a texture-mapping approach. The texture-mapping approach is generally used as a method for putting surface texture into a computer-reconstructed 3D surface model [54]. The 2D photos like natural color photos are available for the surface model reconstruction. Through the coordinate's correspondence of the $2 \mathrm{D}$ image to the $3 \mathrm{D}$ model, the feature of the $2 \mathrm{D}$ image is automatically matched onto each wireframe shell of the 3D model. A rigid choice of the matching points between 2D and 3D images is very important to generate the complicated texture mapping of the paprika. Then, the matching points were selected from unusual points on each stem and leaf; whereafter, exact 3D natural color models were constructed. From the models, some information can be refined, such as plant diameter and height, leaf shapes and area, which are significant in plant detection and recognition.

\subsection{D Model Measurement of Nursery Plants}

In order to estimate the parameters of the plant from the 3D model, we choose the two clearly-distinguished Rubik's cubes on two sides of the nursery plant box as references to set the referenced coordinates' points because of their standard 3D structure. The length of each edge has a fixed value of $66 \mathrm{~mm}$. The coordinates in one of the two cubes were set as reference coordinates with three-dimensional coordinates $(X, Y, Z)$. These georeferenced point clouds can be used to provide very specific and exact representations of a close depth scene. The measurement parameters include leaf width, leaf length, stem diameter and stem height. According to the coordinates of the referenced points in the 3D model, they were estimated by calculating the distances of the measured marked points in each leaf of the 3D model. For the measurement of each leaf, the distance between the upper end of the petiole to the leaf apex was calculated as the leaf length along the leaf vein. The distance from one edge to another edge in the middle part of the blade was calculated as the leaf width. As for the measurement of the stem, the stem diameter was calculated in the center of the stem. Stem height was calculated from stem tip to soil surface. In this experiment, 18 leaves and 9 stems were chosen from the 3D model and about 100 points on the 3D model were marked for the measurement in each leaf and stem, as well as the cube reference. For the direct measurement of plant growth parameters, we adopted the destructive method to measure the leaf, because plant leaves have irregular shapes and different states, such as wide or narrow, straight or curly, etc. In the experiment sample pot, there were 
9 paprika plants in total. We selected two sample leaves from each paprika plant. We cut and measured them by a ruler. The measurement method for every leaf and stem is the same as the above. Finally, from leaf width and length to stem diameter and height, including spatial summaries of nursery plants, we could obtain the direct and indirect measurement data. According to these data, we could calculate correlation coefficients and Root-Mean-Square Errors (RMSE) between the estimated and measured values of these parameters to obtain their accuracy. We were also able to obtain the percentage of the total number of virtual leaves and stems whose 3D model surface area reconstructed over $95 \%$ or $75 \%$, accounting for the total number of real leaves and stems, to compare the performance of different lenses.

\section{Results}

\subsection{D Modeling Reconstruction of Paprika Plant}

Based on the MSV and SfM algorithms, we have applied the high efficiency MCP-SfM system to obtain many high resolution images from close-up views of four positions. We have respectively tested four types of lenses for one circle of plant shape in four different camera positions, whose focal lengths were $24 \mathrm{~mm}, 28 \mathrm{~mm}, 35 \mathrm{~mm}$ and $50 \mathrm{~mm}$. Table 1 shows that the numbers of acquired images of different lenses in the first and second camera positions, which were on average about 42 photos. However, for the third and fourth camera positions, the number of acquired images of the 50-mm lens is the smallest, because this lens is more sensitive to light intensity in the third and fourth camera positions. Because the MCP-SfM system has been set with certain shooting distance and the indoor light condition, Table 1 demonstrates that the 28-mm lens had good performance while taking photos of nursery plants.

Table 1. The number of images taken by different lenses.

\begin{tabular}{ccccc}
\hline \multirow{2}{*}{ Camera Positions } & \multicolumn{4}{c}{ Focal Length of Lens } \\
\cline { 2 - 5 } & $\mathbf{2 4} \mathbf{~} \mathbf{m m}$ & $\mathbf{2 8} \mathbf{~ m m}$ & $\mathbf{3 5} \mathbf{~} \mathbf{m}$ & $\mathbf{5 0} \mathbf{~ m m}$ \\
\hline 1 & 44 & 44 & 44 & 40 \\
2 & 42 & 41 & 44 & 42 \\
3 & 42 & 43 & 42 & 29 \\
4 & 42 & 45 & 43 & 23 \\
Total & 170 & 173 & 173 & 134 \\
\hline
\end{tabular}

In terms of the resolution and performance of different lenses, we found that $28 \mathrm{~mm}$ was better than other focal lengths. The features of the paprika SfM model are shown in Figure 4.

Figure 4 showed the procedure of 3D modeling for nursery paprika plants reconstructed from a series of photos taken by the 28-mm lens at the first camera position. Firstly, according to the process of 3D modeling, PhotoScan generated point clouds with a total of 88,022 points after registration from the 44 input images captured by the camera, as shown in Figure 4A. The number of points per leaf ranged from 105 to 5140, depending on leaf size. Currently, each point in the clouds of points is determined by two or more images, and overlapping areas have a lot of points. It would be more accurate if this were determined by all possible images, with fewer points, but more precision in the points. It is best to define the minimum number of images for the determination of a point. Secondly, after removing the noise within the point cloud dataset, a total of 1,528,730 dense points remained in Figure 4B, of which 1,528,730 points were classified as shown in gray in Figure 4C. The dense point clouds gave greater precision, and the point number is thirty times more than one of the point clouds and much more accurate. Then, the classified dense clouds of plant leaf were converted into the corresponding polygon wireframe in Figure 4D. It was seen that the polygons consist of many inconsistent triangle wireframes. The complex curves and unevenness on the paprika plant model were generated on the polygon wireframes. The number of polygon wireframes per leaf ranged from 139 to 5765, which was determined by leaf size. 

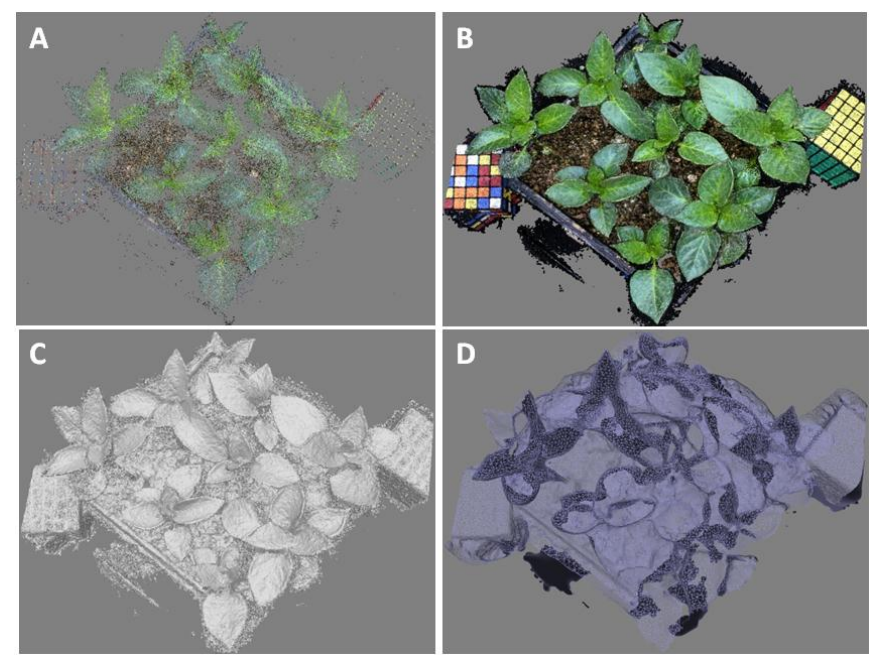

Figure 4. Procedure of 3D modeling for paprika plants reconstructed from a series of photos taken by the 28-mm lens at the first camera position. (A) Point cloud; (B) Dense cloud; (C) Dense cloud classes; (D) Wireframe.

Typically, while building the point clouds, noise also increased with increasing point density. The noise points were a little higher or lower than that expected, which cannot reproduce any actual characteristics within the paprika plants. Besides, the extent of the point cloud dataset was also decreased to minimize processing time, and the point clouds outside of the region were directly classified as noise points. Most of these were removed during building the dense clouds. The filtered point clouds produced an accurate 3D modeling of the paprika plants, as shown in Figure 4. Each leaf or stem feature was clear because of the high-resolution photos captured by the system.

Figure 5 shows the texture mapping of the best 3D models with $0.25-\mathrm{mm}$ resolution for paprika plants by a $28-\mathrm{mm}$ lens in the first and third camera position. In Figure $5 \mathrm{~A}$, leaf models are reconstructed from a series of photos taken by a 28-mm lens at the first camera position. In Figure 5B, leaf and stem models were reconstructed from a series of photos taken by a 28-mm lens at the third camera position. Comparing Figure 5A to Figure 5B, the first camera position had a higher resolution. Moreover, Figure 5 also shows that paprika models in outer pots are generally better than ones in inner pots by the 28 -mm lens in the details. Furthermore, even lower parts of nursery plant shapes can also be estimated and reconstructed. In sum, from Figure 5, we can see that most of the leaf and stem shapes were reconstructed, and their clear models were generated by the 28-mm lens in the first and third camera positions, respectively.
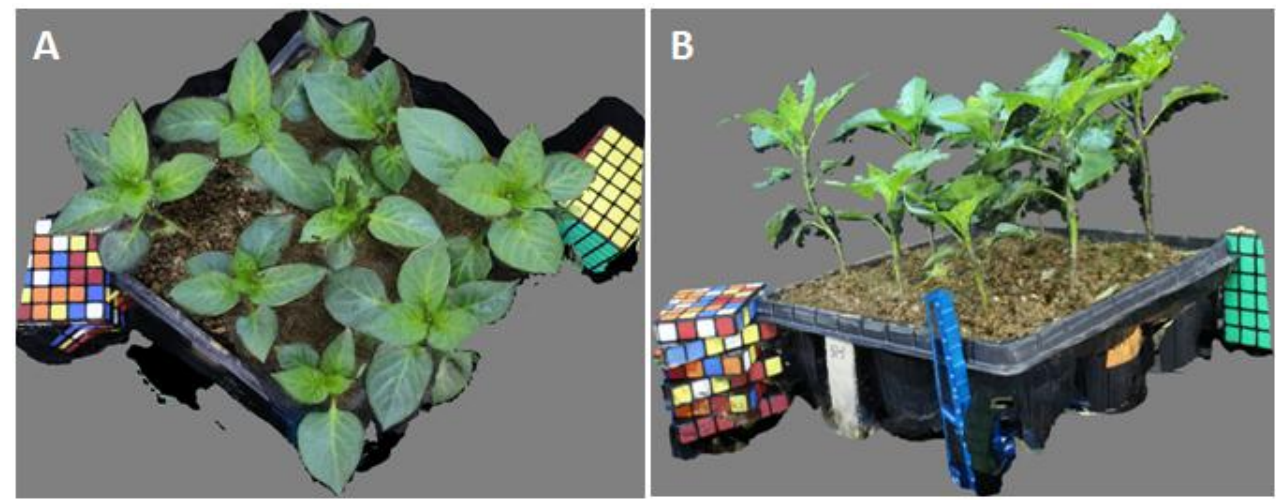

Figure 5. Texture mapping of the best 3D models for paprika plants. (A) Leaf models reconstructed from a series of photos taken by a 28-mm lens at the first camera position; (B) Leaf and stem models reconstructed from a series of photos taken by a 28-mm lens at the third camera position. 


\subsection{Parameters' Estimation of the 3D Paprika Plant Model}

According to the set georeferenced coordinate system and created measurement points in the 3D model, the estimation for leaf and stem shape parameters was conducted. For example, in Figure 5A, the generated leaf lengths ranged between $31 \mathrm{~mm}$ and $64 \mathrm{~mm}$ over all paprika plants. Likewise, in Figure 5B, the generated stem heights ranged between $53 \mathrm{~mm}$ and $96 \mathrm{~mm}$. The difference of nine paprika stems' height between the measured height and the estimated height was found to be less than $1.14 \mathrm{~mm}$. The difference of nine paprika stems' diameter between the measured value and the estimated value was under $0.76 \mathrm{~mm}$. The average difference of stem height and diameter was about $0.6 \mathrm{~mm}$ and $0.31 \mathrm{~mm}$, respectively. Figure 5 demonstrates that the 3D model reconstructed by the 28-mm lens in the first and third camera positions is better than other lenses in other camera positions and camera position groups.

Table 2 illustrates the percentage of the total number of virtual leaves and stems whose 3D model shape surface area reconstructed over 95\% (Category A) or 75\% (Category B) accounting for the total number of real leaves and stems. Ninety five percent and $75 \%$ mean the percentage of the reconstructed surface area of each leaf and stem shape compared to the real shape in the 3D model. Category A means the percentage of the 3D model shape surface area reconstructed over 95\% compared to the real shape for both leaves and stems. Category B means the percentage of the 3D model shape surface area reconstructed over $75 \%$ compared to the real shape for both leaves and stems. The values in Table 2 were obtained by calculating the total number of virtual leaves and stems whose 3D model shape surface area reconstructed over $95 \%$ or $75 \%$ divided by the total number of real leaves and stems in the pot. For example, the total number of real plant leaves is 74. For the 28-mm lens in the first camera position, the total number of virtual leaves whose 3D leaf model shape surface area reconstructed over $95 \%$ is 35 . Therefore, by dividing 35 by 74 , the approximate value of 0.4729 can be obtained. Hence, the percentage of the total number of virtual leaves whose 3D model shape surface area reconstructed over $95 \%$ accounting for the total number of real leaves is about $47 \%$, as seen in Figure 5A. Other values for leaves in Table 2 were obtained in the same way. In other camera positions or camera position groups, the percentage of the total number of virtual leaves whose 3D model shape surface area reconstructed over $95 \%$ accounting for the total number of real leaves is mostly less than $40 \%$. Likewise, the values for stems in Table 2 can also be obtained in the same way. As can be seen in Table 2, for the 28-mm lens in the third camera position, the percentage of the total number of virtual stems whose 3D model shape surface area reconstructed over $95 \%$ accounting for the total number of real leaves is about $100 \%$. Therefore, all of the stem shapes were reconstructed by the $28-\mathrm{mm}$ lens in the third camera position, as seen in Figure 5B. However, in other camera positions or camera position groups, the percentage of the total number of virtual leaves whose 3D model shape surface area reconstructed over $95 \%$ accounting for the total number of real leaves is mostly less than $90 \%$. Thus, from Table 2, we can see that the 28-mm lens in the first and third camera position has great performance in reconstructing the 3D model of the plant.

In general, according to the parameters' estimation result of the 3D paprika plant model, we can analyze the performance of different lens types in different camera positions. These analyses are very helpful for understanding the monitoring of plant growth parameters and the environment, such as light conditions and disease detection. 
Table 2. Percentage of the total number of virtual leaves and stems whose 3D model shape surface area reconstructed over 95\% (Category A) or 75\% (Category B) accounting for the total number of real leaves and stems.

\begin{tabular}{|c|c|c|c|c|c|c|c|c|c|c|c|c|c|c|c|c|}
\hline \multirow{5}{*}{$\begin{array}{l}\text { Camera Positions } \\
\text { (see Figure 1) }\end{array}$} & \multicolumn{8}{|c|}{ Percentage of the Number of Category A or B to That of All Leaves $(\%) * 1$} & \multicolumn{8}{|c|}{ Percentage of the Number of Category A or B to That of All Stems $(\%) * 2$} \\
\hline & \multicolumn{4}{|c|}{$A^{* 3}$} & \multicolumn{4}{|c|}{$B * 4$} & \multicolumn{4}{|c|}{$\mathbf{A}^{* 3}$} & \multicolumn{4}{|c|}{$B * 4$} \\
\hline & \multicolumn{4}{|c|}{$>95$} & \multicolumn{4}{|c|}{$>75$} & \multicolumn{4}{|c|}{$>95$} & \multicolumn{4}{|c|}{$>75$} \\
\hline & \multicolumn{16}{|c|}{ Focal Length of Lens (mm) } \\
\hline & 24 & 28 & 35 & 50 & 24 & 28 & 35 & 50 & 24 & 28 & 35 & 50 & 24 & 28 & 35 & 50 \\
\hline 1 & 34 & 47 & 31 & 32 & 57 & 76 & 74 & 62 & 0 & 0 & 0 & 0 & 11 & 11 & 11 & 0 \\
\hline 2 & 20 & 27 & 27 & 22 & 54 & 59 & 64 & 68 & 44 & 57 & 44 & 33 & 67 & 78 & 78 & 67 \\
\hline $1+2$ & 39 & 45 & 28 & 22 & 59 & 68 & 66 & 64 & 56 & 78 & 44 & 33 & 67 & 78 & 56 & 56 \\
\hline 3 & 3 & 5 & 1 & 0 & 11 & 24 & 9 & 5 & 78 & 100 & 100 & 33 & 89 & 100 & 100 & 44 \\
\hline $1+2+3$ & 9 & 26 & 27 & 0 & 49 & 50 & 69 & 0 & 78 & 89 & 33 & 0 & 89 & 89 & 56 & 0 \\
\hline 4 & 4 & 5 & 4 & 3 & 14 & 19 & 22 & 5 & 44 & 56 & 44 & 22 & 56 & 56 & 78 & 44 \\
\hline $1+2+3+4$ & 7 & 14 & 9 & 16 & 42 & 46 & 41 & 68 & 89 & 89 & 22 & 33 & 89 & 100 & 44 & 44 \\
\hline
\end{tabular}

${ }^{* 1}$ The total number of real plant leaves is $74 ;{ }^{* 2}$ The total number of real plant stems is $9 ;{ }^{* 3}$ Category A means the percentage of 3D model shape surface area reconstructed over $95 \%$

compared to the real shape; ${ }^{* 4}$ Category B means the percentage of $3 \mathrm{D}$ model shape surface area reconstructed over $75 \%$ compared to the real shape. 


\subsection{Error Measurement of the 3D Paprika Plant Model}

In our experiment, the error analyses of the estimated parameters, such as the correlation coefficient and RMSE, were conducted for different lens types and camera positions. According to the error analyses' comparison, the results of the 28-mm lens were demonstrated to be the best. Some models generated by other lenses are not so good, with more noise and calibration errors in some parts of the 3D model. For example, the absolute error of the No. 1 paprika stem height for nine paprika stems is $0.792 \mathrm{~mm}$. Its relative error is 0.008 , which is obtained from 0.792 divided by 99 (measured value). The absolute error of height for nine paprika stems is under $0.2 \mathrm{~mm}$.

Figure 6 shows the coefficient of determination $\left(R^{2}\right)$ and the regression results of leaf and stem for the $28-\mathrm{mm}$ lens. The measured and estimated values were showed in a one-to-one relationship and a regression fit trend line. This demonstrated the accuracy of the best 3D models for leaf and stem shapes of paprika plants taken by the 28 -mm lens. In Figure $6 \mathrm{~A}$, for the leaf length and width of plants taken at the first camera position, the correlation coefficient between the measured and estimated values showed a good fit. Likewise, in Figure 6B, for stem heights and the diameters of plants taken at the third camera position, the regression fit between the measured and estimated values was higher, giving an $R^{2}$ of approximately 1.0. Besides, the spatial resolution was $0.25 \mathrm{~mm}$ per pixel on the $Y$-axis and $0.329 \mathrm{~mm}$ per pixel on the $Z$-axis. The accuracy assessment is high with a mean difference of the $Z$-values of $0.6 \mathrm{~mm}$. Therefore, the accuracy of the 3D model by our MCP system is reliable.
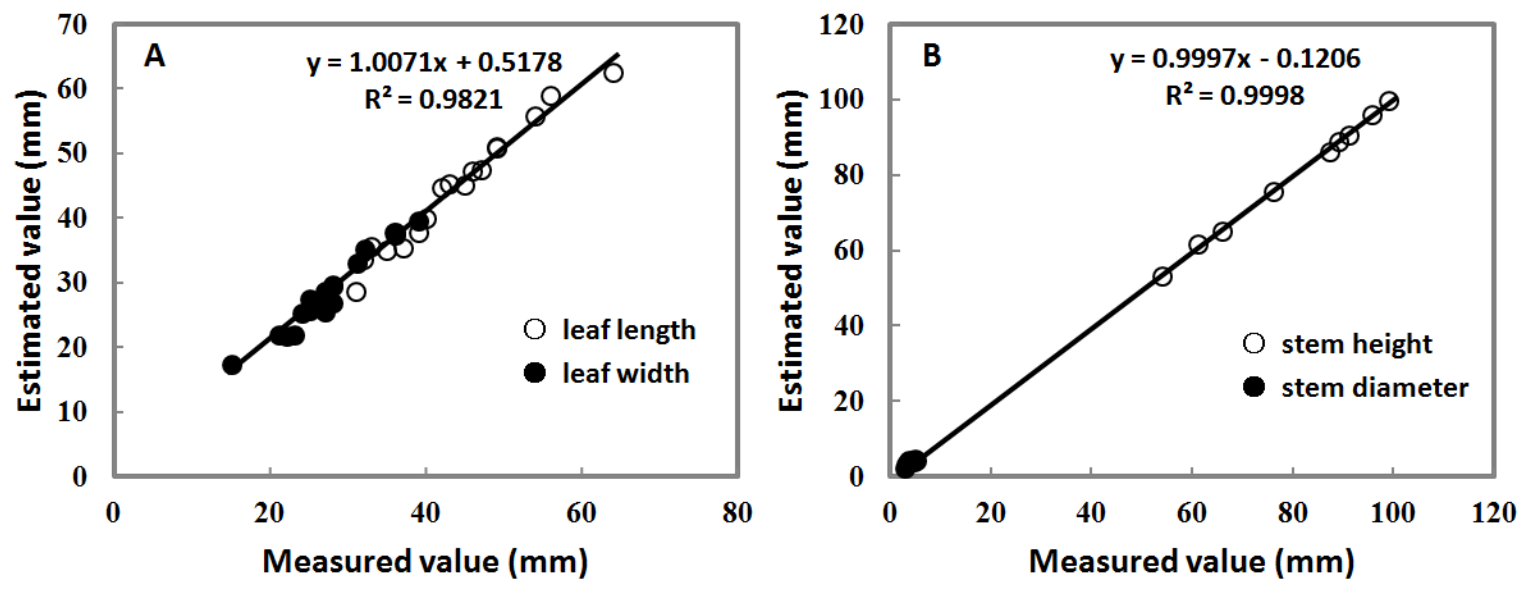

Figure 6. The accuracy of the best 3D models for leaf and stem shapes of paprika plants taken by the 28-mm lens. (A) Leaf length and width of plants taken at the first camera position; (B) Stem heights and diameters of plants taken at the third camera position.

In addition, the RSME of the errors was computed along with the regression fit degree and $\mathrm{R}^{2}$ between measured and estimated parameters. As can be seen in Table 3, the error results of the 3D model of best leaf and stem taken by the 28-mm lens are illustrated. The $R^{2}$ and RMSE of paprika leaf and stem were presented for the whole experiment according to the measurement technique and method. In the first camera position, it showed a correlation coefficient of $0.9821(n=18)$ between the measured and estimated values with a mean difference of $1.65 \mathrm{~mm}$. Likewise, in the third camera position, the RMSE of stem was $1.65 \mathrm{~mm}$, and it showed a correlation coefficient of $0.9998(n=9)$ between the measured and estimated values with a mean difference of $0.57 \mathrm{~mm}$. Besides, Table 3 shows better accuracy of estimation for stem than for leaf in the case of the 28-mm lens. The estimation difference between paprika stem and leaf was because of the limitations of the remote sensing method.

All in all, the above results demonstrated a general consistency between the estimated and measured values for 3D plant model reconstruction by analyzing the statistically-significant differences among different lens types, pointing out the promising role of the MCP system with several consumer-grade cameras for the purposes of plant growth monitoring. 
Table 3. The error results of the 3D model of the best leaf and stem taken by the $28-\mathrm{mm}$ lens.

\begin{tabular}{ccccc}
\hline \multirow{2}{*}{ Camera Positions } & \multicolumn{2}{c}{ Leaf } & \multicolumn{2}{c}{ Stem } \\
\cline { 2 - 5 } & $\mathbf{R}^{\mathbf{2}}$ & RMSE (mm) & $\mathbf{R}^{\mathbf{2}}$ & RMSE (mm) \\
\hline 1 & 0.9821 & 1.65 & & \\
3 & & & 0.9998 & 0.57 \\
\hline
\end{tabular}

\section{Discussions and Conclusions}

\subsection{Advantages of the MCP System Based on the MVS and SfM Methods}

This study presented an evaluation of the accuracy of the 3D image model derived by the MCP-SfM system based on the MVS and SfM technologies. The techniques are usually based on the consistency measurement, where the 3D model is consistent with the input images. They provide viable methods for generating 3D models of vegetation and scenes. The MVS method is a common application of 3D modeling of nursery plants. The SfM method is used to generate 3D models for triangulated meshing and surface reconstruction. These models generated by different lenses or camera positions were compared and evaluated. In general, the SfM method has a great potential in remote sensing range data capture of natural and man-made landscapes to provide very detailed and precise representations. Some small experiments indoors, like the one presented in this study, can be easily monitored using the system. Producing multi-temporal datasets of the entire growth period is essential for obtaining reliable results in such experiments. In the present study, we measured the performance of the highly effective and low cost systems with digital cameras and image reconstruction techniques for estimating the four variables of paprika plants, such as leaf width and length and stem height and diameter. The results showed the practicability of our new method for an exact measurement of plant parameters. The system can make the construction and acquisition of images faster and generate surface models from more viewing angles. The system is also available for extension into real-time monitoring of plant growth in the future. Therefore, it is an ideal system platform for taking more high resolution $3 \mathrm{D}$ images of plants.

\subsection{Error Assessment of the 3D Paprika Plant Model}

For the MCP-SfM system, the challenge is the performance and accuracy of the SfM algorithm and the accuracy of 3D depth data with the effect of ambient illumination. In fact, apart from the error analysis of the 3D paprika plant model, the accuracy of depth data is usually divided between the absolute and relative errors. The absolute error is obtained by the comparison with a known reference value. In the presented case, the absolute error is obtained by comparing the estimated value to a measured one. The relative error can be estimated by calculating the absolute error (the difference between the estimated value and the measured value) divided by the measured value. It gives an indication of how good a measurement is relative to the size of the object being measured. Therefore, the comparative accuracy of these measurements can be determined by looking at their relative errors. Besides, the relative error in the parameters' measurement can better reflect the reliability of measurement accuracy. In particular, this could be considered as one of the reliable evaluation tools in future Digital Elevation Model (DEM) studies.

The precisions achieved on the individual paprika displayed variations determined by the type of the lens and the position of the camera. According to our various experimental comparisons, a focal length larger than $35 \mathrm{~mm}$ could not better reconstruct high resolution 3D models because the measurement devices were installed in a limited scope for indoor conditions. Besides, the upper camera positions were better for the measurement purposes of leaf shape in the 3D model, whereas the lower camera positions are better for the measurement purposes of stem shape. The camera position groups were better for the measurement purposes of the whole plant shape in the 3D model. In general, the performance was the best using the 28-mm lens in the first and third camera positions. According 
to Table 3, the RMSE result of these errors was considered an acceptable error range for estimating the features in consideration of the possible errors' magnitude during manual measurements of the parameters, which could give the validity of the reference data [55,56]. Errors were slightly higher for other lenses or camera positions. What is more, in the above studies, we also found low correlations between the estimated and measured variables, which showed the influence of some limitations of this condition.

In addition, according to the percentage of the total number of virtual leaves and stems whose $3 \mathrm{D}$ model shape surface area reconstructed over $95 \%$ or $75 \%$ accounting for the total number of real leaves and stems (Table 2), the errors for the reconstructed 3D model are obvious among different focal lengths and camera positions. The main reasons for the errors in the parameters' measurement might be not only different overlap rates due to different captured image numbers, but also different exposure rates due to some particularities of the ambient illumination condition. Another reason is the distance between paprika plants and the camera lens in the indoor experimental site, because some larger focal lengths need a longer distance, such as $50 \mathrm{~mm}$ and $85 \mathrm{~mm}$. Thus, it is important for higher 3D model accuracy to adjust the suitable ambient illumination condition and shooting distance in the limited lab scope based on the lighting conditions of different camera positions and the characteristics of different lenses.

\subsection{Limitations and Issues of the Experiment}

In the construction of point clouds, the noises faced in some areas with a complex structure result in sparse black patches, which need to be well represented. According to our investigation, there are some limitations of the imaging system combined with environmental factors. One of the most important factors is light. Generally, lighting should be diffused to reduce errors. Under indoor conditions, the background and shading arrangements have been used to cater to the experiments. In this way, the effect of ambient illumination for the camera varies with the type of lens used. As the quantity, resolution and luminosity of images captured from the cameras of different views are different, there are some differences among different 3D models. In this study, we have discussed the exposure of the camera for the 3D imaging system, which is robust to variation in ambient illumination. It is necessary to compare and discuss the effect of lighting variations under different conditions on the plant canopies in the future. Besides, according to the different quantity of images and the performance of different computers, the entire workflow for producing 3D models from MCP-SfM images requires different time. For stereo images with dimensions of $5184 \times 3456$, it is important to arrange a uniform-luminance environment and a high-performance computer. Keeping the above limitations aside, it is also necessary for further research in the close range stereo imaging of plants to particularly take into account color distortion due to the lighting, sensors and exposure control, as discussed by Nielsen et al. [57]. Moreover, there are inherent limitations of the stereo matching process in this system, which is not robust to all sorts of surfaces and objects expected in agricultural scenarios. This reduces the effect of stereovision and limits either the range or the scale of the application. Finally, other issues with the system are the high performance requirement for the CPU and the large memory needed, especially when we try to do 3D reconstruction at a large scale.

\subsection{Future Improvements and Applications}

According to the above-mentioned issues, some improvements should be made in the future. In order to ensure that the camera in nadir position can take high quality images while capturing images, an adjustable light needs to be fixed for increased resolution. Additionally, we will further propose a method for obtaining the most suitable camera configurations for 3D imaging under different illumination conditions based on observing the precision trends in the camera and lens. Besides, an electrical trigger will also be used to make image acquisition more reliable compared to a mechanical shutter. Meanwhile, taking other sources of error into account, like the inaccuracies caused by rotating plants during data acquisition, the overlap of $90 \%$ among the images will be increased for higher 
accuracy in the future. Different settings were tested in PhotoScan showing that the model quality increased with the number of photos used for model generation. This is also stated by Roberts et al. [58]. Thus, in order to enable plant growth monitoring with higher accuracy, the number of photos taken during the illumination will be increased with a greater variety of viewing perspectives in the future. According to our next design, six more cameras will be installed in the system. Moreover, in the future, the models will also be expected to detect the differences between growths according to the cultivar and treatment.

Acknowledgments: We gratefully acknowledge Parinaz Rahimzadeh-Bajgiran and Hayashi Masao for their kind support.

Author Contributions: K.O. and Y.Z. conceived and designed the experiments; Y.Z. performed the experiments, analyzed the data and wrote the paper; K.O., P.T., F.H., and Y.S. contributed material, analysis tools, discussions and paper revision.

Conflicts of Interest: The authors declare no conflict of interest.

\section{Abbreviations}

The following abbreviations are used in this manuscript:

$\begin{array}{ll}\text { MCP } & \text { Multi-Camera Photography } \\ \text { MVS } & \text { Multiple-View Stereovision } \\ \text { SfM } & \text { Structure from Motion } \\ \text { RMSE } & \text { Root-Mean-Square Error } \\ \text { SIFT } & \text { Scale-Invariant Feature Transform } \\ \text { PPF } & \text { Photosynthesis Photon Flux } \\ \text { PnP } & \text { Perspective N-Point } \\ \text { SSD } & \text { Sum of Squared Differences } \\ \text { DEM } & \text { Digital Elevation Model }\end{array}$

\section{References}

1. Muller-Linow, M.; Pinto-Espinosa, F.; Scharr, H.; Rascher, U. The leaf angle distribution of natural plant populations: Assessing the canopy with a novel software tool. Plant Methods 2015, 11, 1-16. [CrossRef] [PubMed]

2. Omasa, K.; Hosoi, F.; Konishi, A. 3D lidar imaging for detecting and understanding plant responses and canopy structure. J. Exp. Bot. 2007, 58, 881-898. [CrossRef] [PubMed]

3. Spalding, E.P.; Miller, N.D. Image analysis is driving a renaissance in growth measurement. Plant Biol. 2013, 16, 100-104. [CrossRef] [PubMed]

4. Omasa, K.; Takayama, K. Simultaneous measurement of stomatal conductance, non-photochemical quenching, and photochemical yield of photosystem II in intact leaves by yhermal and chlorophyll fluorescence imaging. Plant Cell Physiol. 2003, 44, 1290-1300. [CrossRef] [PubMed]

5. Omasa, K. Image Analysis of Chlorophyll Fluorescence Transients of Cultured Carrot Tissues. Environ. Control Biol. 1992, 30, 127-131. [CrossRef]

6. Omasa, K. Image Instrumentation Methods of Plant Analysis. In Modern Methods of Plant Analysis; Linskens, H.F., Jackson, J.F., Eds.; New Series, Physical Methods in Plant Sciences; Springer-Verlag: Berlin, Germany, 1990; Volume 11, pp. 203-243.

7. Omasa, K.; Takayama, K.; Goto, E. Image Diagnosis of Photosynthetic Injuries Induced by Herbicide in Plants-Comparison of the Induction Method with the Saturation Pulse Method for Chlorophyll a Fluorescence Analysis. J. Soc. High Technol. Agric. 2001, 13, 29-37. [CrossRef]

8. Barbagallo, R.P.; Oxborough, K.; Pallett, K.E.; Baker, N.R. Rapid, noninvasive screening for perturbations of metabolism and plant growth using chlorophyll fluorescence imaging. Plant Physiol. 2003, 132, 485-493. [CrossRef] [PubMed]

9. Garcia, R.; Francisco, J.; Sankaran, S.; Maja, J. Comparison of two aerial imaging platforms for identification of Huanglongbing-infected citrus trees. Comput. Electron. Agric. 2013, 91, 106-115. [CrossRef] 
10. Nuske, S.; Achar, S.; Bates, T.; Narasimhan, S.; Singh, S. Yield estimation in vineyards by visual grape detection. In Proceedings of the IEEE/RSJ International Conference on Intelligent Robots and Systems, San Francisco, CA, USA, 25-30 September 2011; pp. 2352-2358.

11. Astrand, B.; Baerveldt, A. Plant recognition and localization using context information. In Proceedings of the IEEE Conference Mechatronics and Robotics, Aachen, Germany, 13-15 September 2004.

12. Li, L.; Zhang, Q.; Huang, D. A Review of Imaging Techniques for Plant Phenotyping. Sensors 2014, 14, 20078-20111. [CrossRef] [PubMed]

13. Lee, W.S. Sensing technologies for precision specialty crop production. Comput. Electr. Agric. 2010, 74, 2-33. [CrossRef]

14. Preuksakarn, C.; Boudon, F.; Ferrao, F.; Durand, J.-B.; Nikinmaa, E.; Godin, C. Reconstructing plant architecture from 3D laser scanner data. In Proceedings of the 6th International Workshop on Functional-Structural Plant Models, Davis, CA, USA, 12-17 September 2010; pp. 1999-2001.

15. Takizawa, H.; Ezaki, N.; Mizuno, S.; Yamamoto, S. Plant Recognition by Integrating Color and Range Data Obtained Through Stereo Vision. J. Adv. Comput. Intell. Intelli. Inform. 2005, 9, 630-636.

16. Hosoi, F.; Omasa, K. Estimating vertical plant area density profile and growth parameters of a wheat canopy at different growth stages using three-dimensional portable lidar imaging. ISPRS J. Photogramm. Remote Sens. 2009, 64, 151-158. [CrossRef]

17. Hosoi, F.; Nakabayashi, K.; Omasa, K. 3-D Modeling of tomato canopies using a high-resolution portable scanning lidar for extracting structural information. Sensors 2011, 11, 2166-2174. [CrossRef] [PubMed]

18. Nielsen, M.; Andersen, H.; Granum, E. Comparative Study of Disparity Estimations with Multi-Camera Configurations in Relation to Descriptive Parameters of Complex Biological Objects. In Proceedings of the ISPRS Workshop BenCOS 2005: Towards Benchmarking Automated Calibration, Orientation and Surface Reconstruction from Images, Beijing, China, 15 October 2005.

19. Hosoi, F.; Omasa, K. Estimating leaf inclination angle distribution of broad-leaved trees in each part of the canopies by a high-resolution portable scanning lidar. J. Agric. Meteorol. 2015, 71, 136-141. [CrossRef]

20. Endres, F.; Hess, J.; Sturm, J.; Cremers, D.; Burgard, W. 3D Mapping with an RGB-D Camera. IEEE Trans. Robot. 2014, 30, 177-187. [CrossRef]

21. Khoshelham, K.; Elberink, S.O. Accuracy and resolution of kinect depth data for indoor mapping applications. Sensors 2012, 12, 1437-1454. [CrossRef] [PubMed]

22. Omasa, K.; Kouda, M.; Ohtani, Y. 3D microscopic measurement of seedlings using a shape-from-focus method. Trans. Soc. Instrum. Control Eng. 1997, 33, 752-758. [CrossRef]

23. Mizuno, S.; Noda, K.; Ezaki, N.; Takizawa, H.; Yamamoto, S. Detection of Wilt by Analyzing Color and Stereo Vision Data of Plant. In Computer Vision/Computer Graphics Collaboration Techniques of Lecture Notes in Computer Science; Gagalowicz, A., Philips, W., Eds.; Springer Berlin Heidelberg: Berlin, Germany, 2007; Volume 4418, pp. 400-411.

24. Scharstein, D.; Szeliski, R. A Taxonomy and Evaluation of Dense Two-Frame Stereo Correspondence Algorithms. Int. J. Comput. Vis. 2002, 47, 7-42. [CrossRef]

25. Kyto, M.; Nuutinen, M.; Oittinen, P. Method for measuring stereo camera depth accuracy based on stereoscopic vision. Proc. SPIE 2011, 7864. [CrossRef]

26. Van der Heijden, G.; Song, Y.; Horgan, G.; Polder, G.; Dieleman, A.; Bink, M.; Palloix, A.; van Eeuwijk, F.; Glasbey, C. SPICY: Towards automated phenotyping of large pepper plants in the greenhouse. Funct. Plant Biol. 2012, 39, 870-877. [CrossRef]

27. Biskup, B.; Scharr, H.; Schurr, U.; Rascher, U. A stereo imaging system for measuring structural parameters of plant canopies. Plant Cell Environ. 2007, 30, 1299-1308. [CrossRef] [PubMed]

28. Kise, M.; Zhang, Q. Development of a stereovision sensing system for 3D crop row structure mapping and tractor guidance. Biosyst. Eng. 2008, 101, 191-198. [CrossRef]

29. Ivanov, N.; Boissard, P.; Chapron, M.; Valery, P. Estimation of the height and angles of orientation of the upper leaves in the maize canopy using stereovision. Agronomie 1994, 14, 183-194. [CrossRef]

30. Kazmi, W.; Foix, S.; Alenya, G.; Andersen, H.J. Indoor and outdoor depth imaging of leaves with time of flight and stereo vision sensors: Analysis and comparison. ISPRS J. Photogramm. Remote Sens. 2014, 88, 128-146. [CrossRef]

31. Okutomi, M.; Kanade, T. A multiple-baseline stereo. IEEE Trans. Pattern Anal. Mach. Intell. 1993, 15, 353-363. [CrossRef] 
32. Vogiatzis, G.; Hernández, C.; Torr, P.H.S.; Cipolla, R. Multiview stereo via volumetric graph-cuts and occlusion robust photoconsistency. IEEE Trans. Pattern Anal. Mach. Intell. 2007, 29, 2241-2246. [CrossRef] [PubMed]

33. Vu, H.-H.; Labatut, P.; Keriven, R.; Pons, J.P. High accuracy and visibility-consistent dense multi-view stereo. IEEE Trans. Pattern Anal. Mach. Intell. 2012, 34, 889-901.

34. Harwin, S.; Lucieer, A. Assessing the Accuracy of Georeferenced Point Clouds Produced via Multi-View Stereopsis from Unmanned Aerial Vehicle (UAV) Imagery. Remote Sens. 2012, 4, 1573-1599. [CrossRef]

35. Fonstad, M.A.; Dietrich, J.T.; Courville, B.C.; Jensen, J.L.; Carbonneau, P.E. Topographic structure from motion: A new development in photogrammetric measurement. Earth Surf. Proc. Landf. 2013, 38, 421-430. [CrossRef]

36. Lowe, D. Distinctive image features from scale-invariant key points. Int. J. Comput. Vis. 2004, 60, 91-110. [CrossRef]

37. Snavely, N.; Seitz, S.M.; Szeliski, R. Modeling the world from internet photo collections. Int. J. Comput. Vis. 2008, 80, 189-210. [CrossRef]

38. Mathews, A.J.; Jensen, J.L.R. Three-dimensional building modeling using structure from motion: improving model results with telescopic pole aerial photography. In Proceedings of the 35th Applied Geography Conference, Minneapolis, MN, USA, 10-12 October 2012; pp. 98-107.

39. Pollefeys, M.; Gool, L.V.; Vergauwen, M.; Verbiest, F.; Cornelis, K.; Tops, J. Visual modeling with a hand-held camera. Int. J. Comput. Vis. 2004, 59, 207-232. [CrossRef]

40. Leberl, F.; Irschara, A.; Pock, T.; Meixner, P.; Gruber, M.; Scholz, S.; Weichert, A. Point clouds: Lidar versus 3D vision. Photogramm. Eng. Remote Sens. 2010, 76, 1123-1134. [CrossRef]

41. Turner, D.; Lucieer, A.; Watson, C. Development of an unmanned aerial vehicle (UAV) for hyper resolution mapping based visible, multispectral, and thermal imagery. In Proceedings of the 34th International Symposium of Remote Sensing Environment, Sydney, Australia, 10-15 April 2011.

42. Dey, A.; Mummet, L.; Sukthankar, R. Classification of plant structures from uncalibrated image sequences. In Proceedings of the IEEE Workshop on Applications of Computer Vision, Breckenridge, CO, USA, 9-11 January 2012; pp. 9-11.

43. Dandois, J.P.; Ellis, E.C. Remote sensing of vegetation structure using computer vision. Remote Sens. 2010, 2, 1157-1176. [CrossRef]

44. Wilburn, B.; Joshi, N.; Vaish, V.; Talvala, E.; Antunez, E.; Barth, A.; Adams, A.; Levoy, M.; Horowitz, M. High Performance Imaging Using Large Camera Arrays. Trans. Graph. ACM 2005, 24, 765-776. [CrossRef]

45. Popovic, V.; Afshari, H.; Schmid, A.; Leblebici, Y. Real-time implementation of gaussian image blending in a spherical light field camera. In Proceedings of the IEEE International Conference on Industrial Technology, Cape Town, South Africa, 25-28 February 2013.

46. Nguyen, T.T.; Slaughter, D.C.; Max, N.; Maloof, J.N.; Sinha, N. Structured Light-Based 3D Reconstruction System for Plants. Sensors 2015, 15, 18587-18612. [CrossRef] [PubMed]

47. Barone, S.; Paoli, A.; Razionale, A.V. A Coded Structured Light System Based on Primary Color Stripe Projection and Monochrome Imaging. Sensors 2013, 13, 13802-13819. [CrossRef] [PubMed]

48. Mathews, A.J.; Jensen, J.L.R. Visualizing and quantifying vineyard canopy lai using an unmanned aerial vehicle (UAV) collected high density structure from motion point cloud. Remote Sens. 2013, 5, 2164-2183. [CrossRef]

49. Turner, D.; Lucieer, A.; Watson, C. An automated technique for generating georectified mosaics from ultra-high resolution unmanned aerial vehicle (UAV) imagery, based on structure from motion (SfM) point clouds. Remote Sens. 2012, 4, 1392-1410. [CrossRef]

50. Snavely, N. Scene Reconstruction and Visualization from Internet Photo Collections. Ph.D. Thesis, University of Washington, Seattle, WA, USA, 2008.

51. Tomasi, C.; Kanade, T. Shape and motion from image streams under orthography: A factorization method. Int. J. Comput. Vis. 1992, 9, 137-154. [CrossRef]

52. Moreno-Noguer, F.; Lepetit, V.; Fua, P. Accurate Non-Iterative O(n) Solution to the PnP Problem. In Proceedings of the IEEE International Conference on Computer Vision, Rio de Janeiro, Brazil, 14-21 October 2007.

53. Triggs, B.; Mclauchlan, P.; Hartley, R.; Fitzgibbon, A. Bundle adjustment-A modern synthesis. In Vision Algorithms: Theory and Practice; Springer Berlin Heidelberg: Berlin, Germany, 2000; Volume 1883, pp. 298-375. 
54. Koniaris, C.; Cosker, D.; Yang, X.S.; Mitchell, K. Survey of Texture Mapping Techniques for Representing and Rendering Volumetric Mesostructure. J. Comput. Graph. Tech. 2014, 3, 18-60.

55. Paulus, S.; Behmann, J.; Mahlein, A.-K.; Plümer, L.; Kuhlmann, H. Low-Cost 3D Systems: Suitable Tools for Plant Phenotyping. Sensors 2014, 14, 3001-3018. [CrossRef] [PubMed]

56. Paproki, A.; Sirault, X.; Berry, S.; Furbank, R.; Fripp, J. A novel mesh processing based technique for 3D plant analysis. BMC Plant Biol. 2012, 12. [CrossRef] [PubMed]

57. Nielsen, M.; Andersen, H.J.; Slaughter, D.C.; Granum, E. Ground truth evaluation of computer vision based 3D reconstruction of synthesized and real plant images. Precis. Agric. 2007, 8, 49-62. [CrossRef]

58. Roberts, R.; Sinha, S.N.; Szeliski, R.; Steedly, D. Structure from motion for scenes with large duplicate structures. In Proceedings of the Computer Vision and Patter Recognition, Providence, RI, USA, 20-25 June 2011; pp. 3137-3144.

(C) 2016 by the authors; licensee MDPI, Basel, Switzerland. This article is an open access article distributed under the terms and conditions of the Creative Commons Attribution (CC-BY) license (http://creativecommons.org/licenses/by/4.0/). 